\title{
An innovative digital imaging set-up allowing a low-dose approach to phase contrast applications in the medical field
}

\author{
A. Olivo, ${ }^{\text {a) }}$ F. Arfelli, G. Cantatore, and R. Longo \\ Dipartimento di Fisica, Università di Trieste e INFN, Sezione di Trieste, Via Valerio 2, 34100 Trieste, Italy \\ R. H. Menk \\ Sincrotrone Trieste ScPA, Strada Statale 14 Km 163.5, 34100 Trieste, Italy \\ S. Pani, M. Prest, P. Poropat, and L. Rigon \\ Dipartimento di Fisica, Università di Trieste e INFN, Sezione di Trieste, Via Valerio 2, 34100 Trieste, Italy \\ G. Tromba \\ Sincrotrone Trieste ScPA, Strada Statale 14 Km 163.5, 34100 Trieste, Italy \\ E. Vallazza and E. Castelli \\ Dipartimento di Fisica, Università di Trieste e INFN, Sezione di Trieste, Via Valerio 2, 34100 Trieste, Italy
}

(Received 20 November 2000; accepted for publication 30 May 2001)

\begin{abstract}
Recently, new imaging modalities based on the detection of weak phase perturbations effects, among which are phase contrast and diffraction imaging, have been developed by several researchers. Due to their high sensitivity to weakly absorbing details, these techniques seem to be very promising for applications in the medical field. On the other hand, digital radiology is undergoing a wide diffusion, and its benefits are presently very well understood. Up to now, however, the strong pixel size constraints associated with phase contrast pattern detection limited the possibility of exploiting the advantages of phase contrast in digital radiology applications. In this paper, an innovative setup capable of removing the pixel size constraints, and thus opening the way to low dose digital phase contrast imaging, is described. Furthermore, we introduce an imaging technique based on the detection of radiation scattered at small angles: the information extracted from the sample is increased at no dose expense. We believe that several radiological fields, mammography being the first important example, may benefit from the herein described innovative imaging techniques. (C) 2001 American Association of Physicists in Medicine. [DOI: 10.1118/1.1388219]
\end{abstract}

Key words: phase contrast, diffraction, digital radiology, synchrotron radiation, image enhancement

\section{INTRODUCTION}

One of the main problems in diagnostic radiology is the low image contrast due to poor x-ray absorption differences. This limitation is particularly relevant in the field of mammography, where low contrast masses and small size microcalcifications have to be detected. In the first case, relatively large objects having an $\mathrm{x}$-ray absorption coefficient very close to normal tissue are studied; in the second case, the absorption coefficient difference is high (calcium versus soft tissue), but the extremely small dimensions of the calcified structures (hundreds of microns or less) result in very poor intensity differences in the x-ray beam transmitted through the detail and immediately outside it. ${ }^{1-5}$ In some cases, these obstacles result in limitations in terms of sensitivity as well as specificity of the mammographic examinations. ${ }^{6}$

A solution to this problem consists in developing new detectors with extremely high contrast resolution. In this framework, very promising results have been obtained by means of single photon counting detector devices, like the SYRMEP/FRONTRAD (SYnchrotron Radiation for MEdical Physics/FRONTier RADiology) detector prototype, ${ }^{7-10}$ or other similar devices. ${ }^{11}$ The ability of counting every single photon clearly maximizes the contrast resolution in the images, since the only noise source lays in the intrinsic statistic nature of $\mathrm{x}$-ray emission and absorption (quantum noise). ${ }^{12-14}$ This approach, however, while pushing further down the minimum detectable contrast threshold, still relies only on the absorption properties of the imaged object, and thus to some extent it is still subject to the limitations mentioned above.

A completely different strategy is employed by phase contrast imaging, which has recently been deeply investigated by several researchers, especially because of its relevant potentials in the medical field. ${ }^{15-24}$ In the phase contrast approach, image contrast arises from the real part of the refractive index (commonly referred to as $\delta$ in the literature, the refractive index being expressed as $\mathrm{n}=1-\delta+\mathrm{i} \beta$ ), rather than from its imaginary part $(\beta)$. For most materials (and especially for soft tissue), in the energy range of radiology (below $100 \mathrm{keV}$ ), $\delta$ is much larger than $\beta$; consequently, the effects due to $\delta$ are, in general, much more relevant than those due to $\beta$. Contrast formation is due to interference between the wave fronts that have been phase shifted by a detail inside the imaged sample and the unperturbed wave fronts propagating in the immediate vicinity of the detail itself. This interference effect takes place within a very nar- 
row angular region (10-100 $\mu \mathrm{rad})$, and inside this region an interference pattern can be detected. An exhaustive description of the interference pattern formation can be found in Refs. 25-26. The aspect that we want to stress here is the possibility of obtaining a very high image contrast also when imaging extremely low absorbing details, since the involved physical process is completely different and does not rely on $\mathrm{x}$-ray absorption. Ideally, completely nonabsorbing details (i.e., with exactly the same value of $\beta$ as the background in which they are embedded) may also be detected by means of this technique, if their $\delta$ value differs to some extent from the background value.

In order to apply the technique to diagnostic radiology, however, a few relevant problems have to be solved. First of all, since the interference angle is very small, it is impossible to place the detector device immediately behind the imaged sample: the sample-to-detector distance has to be increased in order to convert the small angular interference region into linear dimensions compatible with the detector spatial resolution. On the other hand, the finite source size results in excessive blurring effects (which could smear the interference peaks) if this distance is made too large; thus, a compromise between these counteracting effects has to be found according to the specific imaging requirements (imaged sample characteristics, detector spatial resolution, radiation source dimensions, and so on). An example of sample-todetector distance optimization, based on Fresnel-Kirchoff diffraction integrals evaluation, can be found in Ref. 22. The same reference demonstrates that, if the source size is too large, the phase contrast technique provides very poor image quality. Thus, extremely high spatial coherence sources are required for phase contrast imaging: namely, synchrotron radiation sources or microfocus x-ray tubes. Chromatic coherence is much less critical, and, in fact, phase contrast images have also been obtained by means of polychromatic sources. ${ }^{27}$ Most of the works reported in the references, however, are concerned with synchrotron radiation experiments, since with microfocus $\mathrm{x}$-ray tubes phase contrast imaging requires extremely long exposure times $\left(\sim 2 \mathrm{~h}^{27}\right)$ due to the low emitted flux.

Even when high coherence sources are used, the detection of secondary interference peaks requires detectors with extremely high spatial resolution: due to this fact, the first phase contrast experiments were carried out utilizing high resolution x-ray films or high resolution CCD cameras (see, for instance, Refs. 15-16). The low detection efficiency of these devices, however, prevented clinical applications of the technique, due to the high radiation dose consequently delivered to the samples. More recently, low dose phase contrast images were obtained by means of conventional film-screen systems: ${ }^{21-22}$ it was, in fact, observed that the convolution between the phase contrast patterns and the film-screen system point spread function preserves relevant improvements in the detected signal, especially in terms of edge enhancement. In this framework, effort is now directed toward a digital approach to low dose phase contrast imaging, since it would result in combining the well-known advantages of both phase contrast and digital imaging. In principle, this would require a high efficiency, high spatial resolution (tenths of $\mu \mathrm{m}$ ) detector device; but in most cases detection efficiency and spatial resolution are counteracting issues. Even though some other possibilities might have been investigated (based, for instance, on the optimization of the scintillator coating and of the pixel size of a CCD camera, or on the development of appropriate flat panel devices), our experience with the SYRMEP/FRONTRAD detector prototype encouraged us to search for a possible application of this device in the phase contrast field. Following this strategy, a relevant dose reduction can be provided by the very high detection efficiency $(>80 \%$ at $20 \mathrm{keV})$ of the device, while at the same time the single photon counting capability of the readout electronics allows the detection of very small signals. In order to check the feasibility of this approach, we first reduced the detector pixel size by means of a narrow, fully absorbing slit placed in front of the detector sensitive area. Then, a detailed analysis of the basic physical principles of phase contrast image formation enabled us to develop an innovative setup, which gives the possibility of removing - at least in one dimension-the pixel size constraints, thus achieving low dose, single photon counting, digital phase contrast images.

Finally, it is important to underline that all the images that will be presented below have been obtained at relatively low (17-22 keV) photon energies. In this way, the phase contrast signal (due to $\delta$ ) is superimposed to the conventional absorption signal (due to $\beta$ ), and both contrast sources are exploited at the same time.

Another possibility might consist in highly increasing the beam energy. This would result in a strong reduction of the dose delivered to the sample, but also in an excessive decrease of the absorption contrast with respect to the phase contrast signal (in other words, only the edges of the details would be detected). Our approach thus preserves all the features of conventional absorption imaging, while at the same time the visibility of all details is dramatically enhanced by the phase effects.

\section{MATERIALS AND METHODS}

\section{A. The beamline}

The SYRMEP beamline has been described in detail in previous papers ${ }^{28-29}$ thus here just a few main characteristics will be given. The radiation source results from one of the bending magnets of the storage ring. Source dimensions (i.e., the transverse dimensions of the electron bunches when crossing the magnet) are equal to 1100 (width) $\times 140$ (height) $\mu \mathrm{m}^{2}$ FWHM (full width at half-maximum). The beam is carried to the experimental room, located at approximately $20 \mathrm{~m}$ from the source, through a high vacuum beam pipe. Along the pipe a channel-cut Si $(1,1,1)$ crystal monochromatizes the beam with an energy resolution of about $0.2 \%$ in the energy range $10-35 \mathrm{keV}$. At the entrance of the 
experimental room, a tungsten slit system, moved by micrometric precision stepper motors, allows a precise definition of the beam cross section; the maximum available cross section is 100 (width) $\times 4$ (height, FWHM) $\mathrm{mm}^{2}$. A larger monocromator crystal is currently under development: this device will provide us in the near future a wider beam cross section $\left(150 \times 4 \mathrm{~mm}^{2}\right)$. The experimental room is equipped with a large area ionization chamber, which allows beam monitoring and thus delivered dose evaluation, a sample movement stage, and the detector prototype, which is described in the next section.

\section{B. The detector}

The SYRMEP/FRONTRAD detector prototype is a silicon microstrip detector used in edge-on geometry, i.e., with radiation impinging on the side rather than on the surface of the wafer (see diagram 1). The dimensions of the wafer are

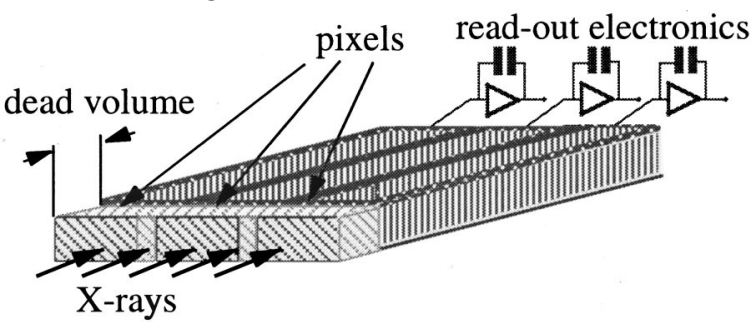

equal to 51.2 (width) $\times 0.3$ (height) $\times 10$ (depth) $\mathrm{mm}^{3}$. On one of the large surfaces of the device 256 parallel strips are implanted, and thus the device is subdivided into 256 independent cells, each one having dimensions equal to 0.2 (width) $\times 0.3$ (height) $\times 10$ (depth) $\mathrm{mm}^{3}$. Therefore, in the edge-on geometry, the incoming photons impinge on an array of 256 pixels, the dimensions of which are determined by the strip pitch $(200 \mu \mathrm{m})$ in the horizontal direction and by the wafer thickness $(300 \mu \mathrm{m})$ in the vertical direction. Since the depth of the single detector cell is $1 \mathrm{~cm}$, photons in the considered energy range $(15-35 \mathrm{keV})$ are nearly completely absorbed within the detector volume. Thus, an absorption efficiency practically of $100 \%$ is obtained. On the other hand, a thin undepleted entrance window is strictly necessary in order to preserve the detector noise performances; the depth of this "dead volume" is of the order of 150-200 $\mu \mathrm{m}$. As a result, the overall detection efficiency of the device is of the order of $80 \%$ at mammographic energies.

The electronic chain reading out the signal, completely realized in VLSI (Very Large Scale Integration) technology, operates in a single photon counting mode. After a chargesensitive preamplifier and several amplifying and shaping stages, a variable threshold discriminator and a scaler enable us to completely cut off the noise and count every single signal higher than the threshold for photon energies higher than $15 \mathrm{keV}$. Thus, only quantum noise is present in the acquired images, and image contrast is consequently maximized.

A more detailed description of the detector prototype and readout electronics can be found in Refs. 7-10.

\section{The imaging setup}

As described in Sec. II, a highly collimated, $100 \times 4 \mathrm{~mm}^{2}$ laminar monochromatic beam is available in the experimental room: this beam is usually reduced down to $\sim 512$ $\times 0.3 \mathrm{~mm}^{2}$ by means of the tungsten slits and kept stationary during image acquisition. The detector prototype discussed in the previous section is mounted on a two-axis micrometric precision positioning stage. In this way, the detector active surface can be carefully matched to the laminar beam cross section. Once this alignment is obtained, the detector is kept stationary with respect to the beam and the two-dimensional images are acquired by vertically scanning the sample through the beam by means of a micrometric precision scanning stage. Further details on the SYRMEP/ FRONTRAD imaging acquisition technique can be found in Refs. 28-30. In order to perform phase contrast imaging it is not possible to place the sample in contact with the detector, but it is rather necessary to optimize the sample-to-detector distance. Following previous calculations ${ }^{22}$ and the simulations described in the next section, the sample scanning stage has been placed at a distance of approximately $1.8 \mathrm{~m}$ from the detector. Since the source-to-detector distance is slightly lower than $25 \mathrm{~m}$, we obtain in this way a source-to-sample distance of approximately $23 \mathrm{~m}$. This distance results in a high spatial coherence in the vertical direction (corresponding to the source dimension equal to $140 \mu \mathrm{m}$ FWHM) and in a lower coherence in the horizontal direction, where the source size is larger. In this paper we basically deal with optimizing the phase contrast image quality in the vertical direction, i.e., the direction along which the sample is scanned through the beam.

Nevertheless, as will be shown in the next section, very high overall image quality has been achieved with the described technique.

\section{The simulations}

Before the experiment was carried out, most of the expected results have been simulated by means of routines specifically developed for the purposes of the present work. In the next section nearly all the experimental results will be presented together with the simulated ones, and, as it will be seen, in all such cases a good agreement between simulation and experiment is obtained.

A precise evaluation of a phase contrast pattern can be made by means of Fresnel-Kirchoff integrals. ${ }^{25-26}$ In this way, the ideal image that would be obtained by illuminating the sample with a point source and by means of an "infinite" spatial resolution detector can be evaluated. Then, the use of a finite size source and of a finite resolution detector has to be taken into account: this can be done by means of convolution integrals. This approach has already been described in Ref. 22.

A simpler-but more approximated-strategy is based on the so-called paraxial approximation: ${ }^{16,27}$ this technique provides the possibility of evaluating the diffraction angle of a photon outcoming from the object by means of very simple 
equations (basically, the deflection angle is proportional to the gradient of $\delta$; see the quoted references for full formulas).

In this way, the path of each photon emitted by the source and crossing the sample can be followed, similarly to what is done in ray tracing. This simulation receives in input the dimensions of the region from which photons are emitted (the source size) and the area they must reach after having crossed the sample in order to be detected (the detector pixel size); thus, it is not required any convolution on the output signal.

Results obtained with this approximated technique have been compared to the ones provided by the more rigorous Fresnel-Kirchoff approach, and a good agreement was found. ${ }^{31}$ This is due to the fact that when Fresnel-Kirchoff patterns are convolved with a source size and a detector pixel that are not very small, all the fine features of the pattern, which constitute the main difference between the two approaches, are lost. Thus, in most practical cases the two techniques give nearly the same results. This aspect will be made clearer when the first simulated patterns will be presented, at the beginning of the following section. As a last remark, it should be noted that by introducing the refractive index as a complex number $(n=1-\delta+i \beta)$ in the simulations, both absorption and phase contrast effects are taken into account in the patterns.

\section{RESULTS AND DISCUSSION}

\section{A. Pixel size constraints}

By means of the simulations described above, we first evaluated the effect of the detector pixel size on the phase contrast signal: the result is shown in Fig. 1. Figure 1(a) shows the "pure" interference pattern that would be obtained by illuminating a $100 \mu \mathrm{m}$ diam nylon wire with a monochromatic (17 keV) point source. Figures 1(b), 1(c), 1(d), and 1(e) show the same pattern acquired by means of a detector pixel having dimensions equal to $25,50,100$, and $300 \mu \mathrm{m}$, respectively. The source-to-sample distance was $23 \mathrm{~m}$ and the sample-to-detector distance was $1.8 \mathrm{~m}$, as occurs in the experimental conditions. As it can be seen, the convolution with the pixel size smears out the phase contrast pattern, and the larger the pixel the smaller the acquired signal. All the patterns presented in Fig. 1 have been obtained by means of the Fresnel-Kirchoff integral technique described above. Nevertheless, the paraxial approximation would have given almost the same results for Figs. 1(c)-1(e) and would have reproduced to a very good approximation also Fig. 1(b). Only the pattern shown in Fig. 1(a) would not be obtainable by means of the paraxial approximation; however, this kind of fine pattern cannot be acquired with our experimental setup.

As it can be seen from Fig. 1(e), very poor phase contrast images would be collected with the SYRMEP/FRONTRAD detector (pixel height $=300 \mu \mathrm{m}$ ) without applying the techniques described in the next sections. The signal in Fig. 1(e) is of the order of $1 \%$, and thus it would not contribute significantly to the detection of details within a sample. Some
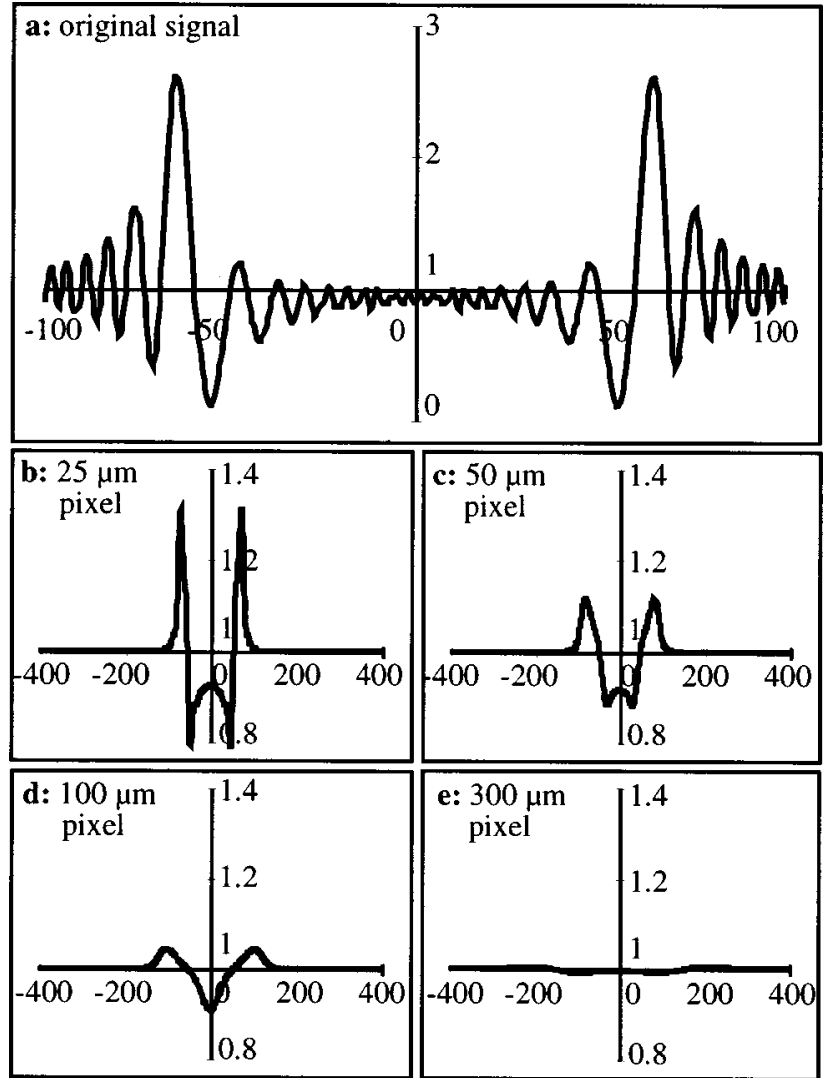

FIG. 1. Influence of the pixel dimensions on the phase contrast signal (see the text). Above: simulated interference pattern obtainable with a point source and a detector with "infinite" spatial resolution. Below: from left to right and top to bottom, the same pattern as it would appear if acquired with a $25,50,100$, and $300 \mu \mathrm{m}$ pixel, respectively. The signal is smeared out as the pixel size is increased. For all graphs, the relative intensity is given as a function of the spatial displacement in microns.

phase contrast images of nylon wires have been acquired with the $300 \mu \mathrm{m}$ detector, and the detected signal was of the order of $1 \%$, in agreement with the predictions of the simulation.

\section{B. Pixel size reduction}

In order to increase the phase contrast signal, it is thus necessary to reduce the pixel dimensions. The first attempt at pixel size reduction consisted in placing a copper mask in front of the detector device. The mask was obtained by carving a $100 \mu \mathrm{m}$ high, $5 \mathrm{~cm}$ long slit into a $0.7 \mathrm{~mm}$ thick copper foil. The thickness of the copper foil is large enough to guarantee full absorption of photons in the considered energy range. Thus, when the slit is perfectly aligned with the center of the active surface of the detector device, the pixel size is effectively reduced from 300 (height) $\times 200$ (width) $\mu \mathrm{m}^{2}$ down to 100 (height) $\times 200$ (width) $\mu \mathrm{m}^{2}$. This fine alignment was obtained by mounting the copper mask on a micrometric precision movement stage. The beam height was, of course, reduced accordingly, in order to avoid useless dose delivery to the samples.

With this set-up, i.e., with a pixel size equal to $100 \mu \mathrm{m}$ in the scanning direction, a signal comparable to the one shown 


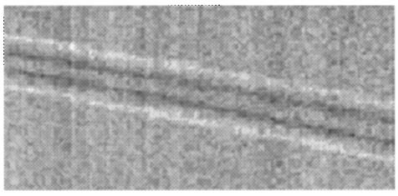

diameter $50 \mu \mathrm{m}$
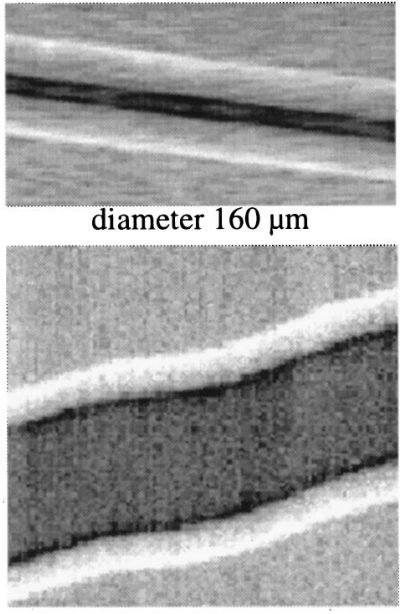

diameter $500 \mu \mathrm{m}$

FIG. 2. Phase contrast images of nylon wires of different diameters obtained by reducing the pixel size from 300 to $100 \mu \mathrm{m}$ by means of a slit (see the text).

in Fig. 1(d) is expected. This signal is of the order of 10\%: a relevant gain should thus be achieved with respect to conventional absorption imaging, which would give for the same object ( $a \sim 100 \mu \mathrm{m}$ thick nylon wire) a signal much lower than $1 \%$ in the energy range of mammography.

A demonstration of this fact is given in Fig. 2, which shows images of three nylon wires (diameters equal to 50, 160, and $500 \mu \mathrm{m}$ ) obtained at $20 \mathrm{keV}$ with this setup: all wires are perfectly detected. In this case, a $10 \mu \mathrm{m}$ scanning step was applied. Figure 3 shows the experimentally measured signal (dots with error bars representing the quantum noise) together with the simulated one (solid line) for all three wires.

In these plots, the relative intensity (i.e., intensity normalized to the background) is reported as a function of distance along the scanning direction; and the signal, i.e., the contrast, can be directly read out on the vertical axis. This signal (measured from peak to peak) is equal to about $4 \%, 8 \%$, and $11.5 \%$ for the 50,160 , and $500 \mu \mathrm{m}$ wire, respectively. Note that the same wires at the same energy would result in a signal of $0.1 \%, 0.3 \%$, and $1 \%$ (respectively) in conventional absorption imaging: thus, a contrast increase of more than one order of magnitude is obtained in all cases by means of the proposed technique.

It should be noted that in this framework the contrast is more significant than the SNR (signal to noise ratio). In fact, since the detector operates in the single photon counting mode, the uncertainty on the number of counts is equal to the square root of the number of counts itself (in all images of Fig. 2, the number of counts per pixel in the background
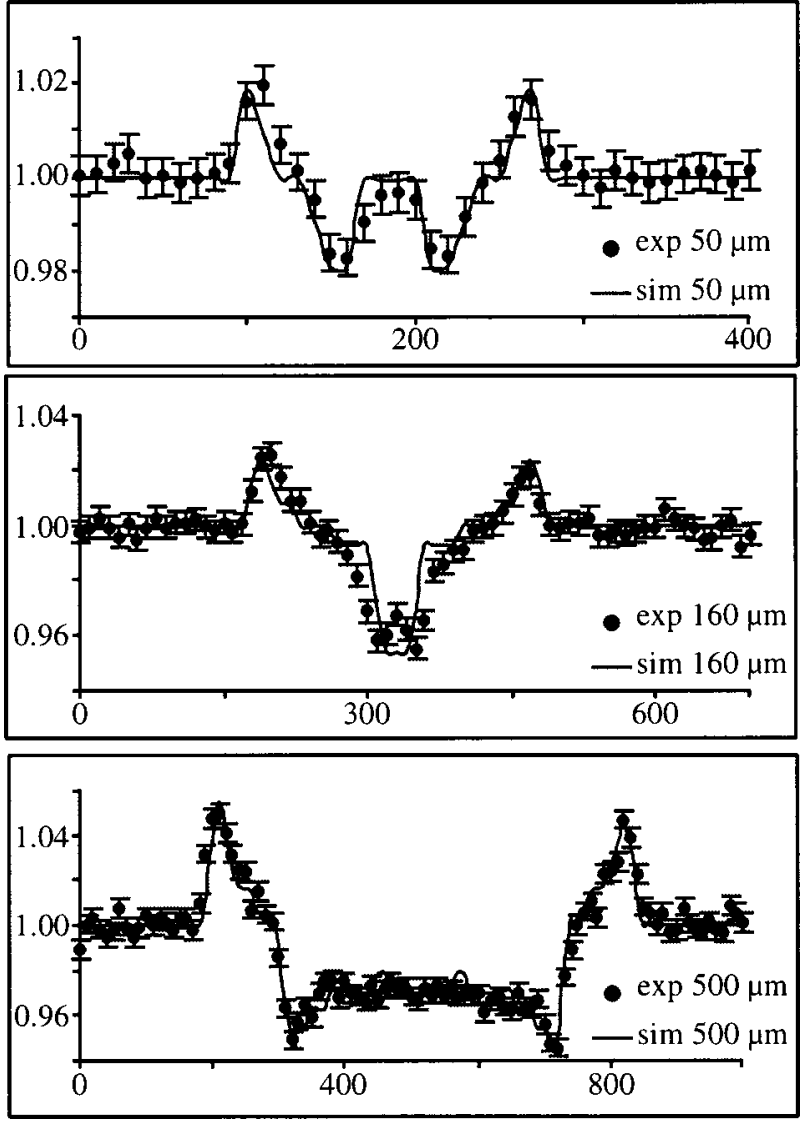

FIG. 3. Experimental data (dots) superimposed on simulated data (solid line) relative to the wires shown in Fig. 2. For all graphs, the relative intensity is given as a function of the displacement in microns. Since the detector operates in a single photon counting mode, the only noise source is due to quantum (Poisson) fluctuations; error bars are thus equal to the square root of the number of counts.

region was approximately 10 000, and thus in Fig. 3 the error bars are of the order of $1 \%$ for all wires).

This means that, given the contrast value, the SNR can be readily evaluated for any photon fluence. Generally speaking, the relevant aspect is the fact that here we are dealing with a physical process that provides increased detail contrast, which is completely different from enhancing the contrast by means of post-processing procedures (unsharp masking, etc.). A low contrast results in a low SNR, unless the delivered dose is dramatically increased, and below some thresholds (Rose criterion, for instance) no post-processing procedure can restore the detail visibility. The possibility of obtaining higher detail contrast in the original images means higher SNR at the same dose or the same SNR at a lower dose, despite any image processing. On the other hand, postprocessing procedures might be applied to phase contrast images to further enhance the detail visibility.

\section{The development of a new setup capable of removing the pixel size constraints}

Even though the image quality that can be seen in Fig. 2 is impressive, this result just demonstrates that phase contrast imaging is feasible if the sample-to-detector distance is op- 


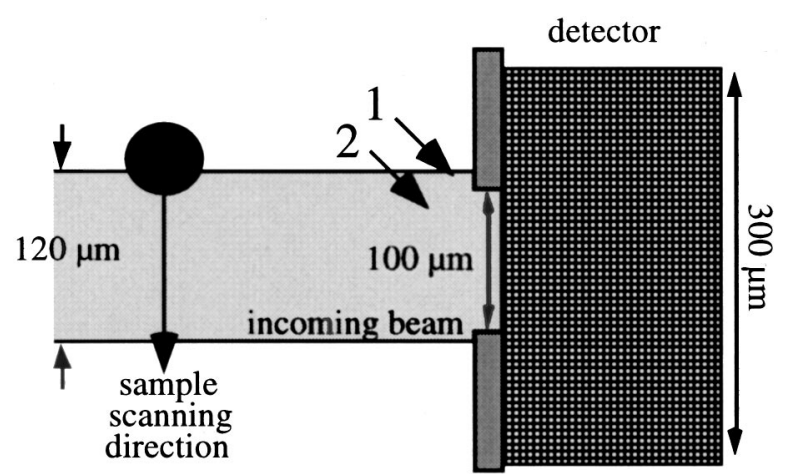

FIG. 4. Schematic of the setup used to acquire the images shown in Fig. 2.

timized, if the source is sufficiently coherent and if the detector pixel is made small enough. Based on these preliminary results, an innovative setup, capable of eliminating the pixel size constraints, has been devised.

First of all, we discuss in deeper detail the setup described in the previous section. It should be noted that, in this configuration, the photons impinging immediately outside the active surface of the pixel when the sample is not present (see the arrow labeled 1 in Fig. 4), although responsible of a slight dose increase with respect to absorption imaging, where sample and detector can be perfectly matched, are of primary importance. When the sample is scanned through the beam, a fraction of those photons is deflected within the active surface of the pixel: this gives rise to the positive peaks in the phase contrast signal. Photons that impinge immediately within the active surface (the arrow labeled 2, which refers to a portion of the beam height approximately comparable to the one falling outside the active surface) have similar importance: when the object passes through the beam, a part of them will be deflected outside the active surface. In this way, the negative peaks in the phase contrast signal are originated. The same situation occurs on the other side of the pixel active area. This somehow implies that photons impinging on the center of the detector active surface are useless: the deviation angle of the photons is, in general, so small ( $\sim 10 \mu \mathrm{rad}$, as quoted above) that those photons will impinge on the detector active surface also after their interaction with the sample. That is to say, those photons just increase the background without a significant contribution to the signal.

This discussion is summarized in Fig. 5, where the dashed line represents the trajectory of the photon without the sample, while the solid line shows the photon deflection (not to scale) due to the passage of the object. On the right-hand side, the resulting (simulated) signal is shown. Three crucial sample positions are shown, and connected by arrows to the corresponding points in the signal; only one-half of the sample scan is schematized, since the situation is symmetric for the remaining part of the scan.

This analysis led us to the development of the second step of the new setup, shown in Fig. 6(a): in this configuration photons in the center of the beam have been eliminated. In this case, the original beam is split in two narrow beams by

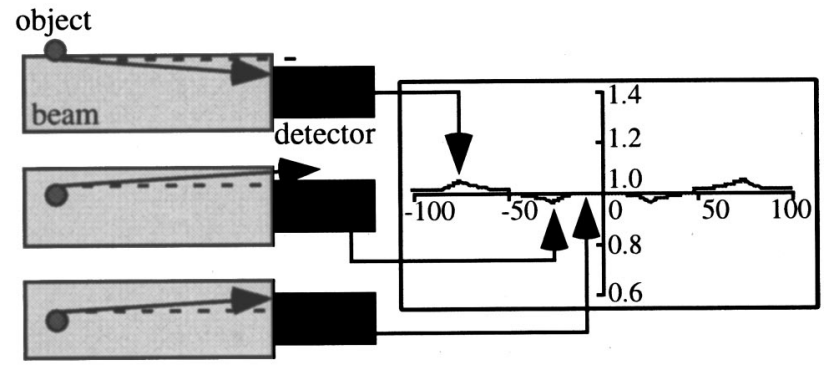

FIG. 5. Schematic of the image formation principle for the images shown in Fig. 2 (see the text), and simulated signal (right) for a $50 \mu \mathrm{m}$ nylon wire placed at $1.8 \mathrm{~m}$ from the detector and illuminated by $20 \mathrm{keV}$ radiation.

a collimator: each narrow beam impinges on one of the detector active area edges. The collimator is placed upstream the sample, in order to avoid unnecessary dose delivery. Comparing the simulated signal shown on the right-hand side of Fig. 6(a) with the one shown in Fig. 5, it is apparent that a relevant gain is acquired by means of this slight change in the set-up. Note that here we are referring to the relative intensity, i.e., the contrast: the discussion is thus independent from dose and/or flux considerations, as discussed in the previous section.

But, apart from the signal increase, it is extremely important to notice that in this configuration the detector pixel size has lost any influence on the intensity of the detected signal: in other words, the extremely stringent (see Fig. 1) pixel size constraints have been eliminated. If the detector pixel now becomes larger, one has to adjust the relative distance between the two beams in such a fashion that they still impinge on the edges of the pixel active area, without changing the height of the beams themselves. In this way, the only change in the signal would be an expansion of the flat region in the middle of the signal. The intensity and the shape of the peaks, however, remain unchanged. Notice that at this point
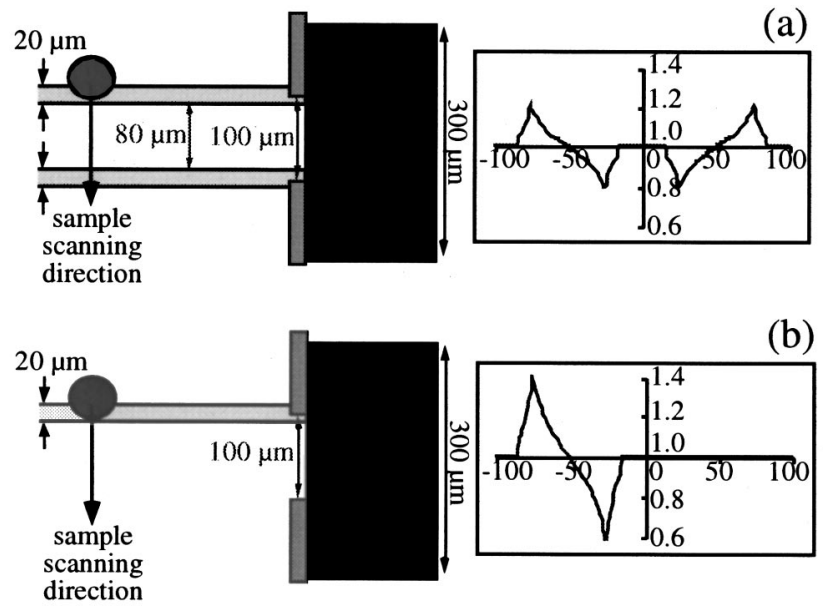

FIG. 6. Experimental setup (left) and simulated signal for a $50 \mu \mathrm{m}$ nylon wire imaged at $20 \mathrm{keV}$ with a sample-to-detector distance of $1.8 \mathrm{~m}$ (right; the relative intensity is plotted as a function of the displacement in microns) for two different imaging techniques. The shown setups provide a relevant contrast increase with respect to the one of Figs. 4-5, and they allow the possibility of removing the pixel size constraints (see the text). 

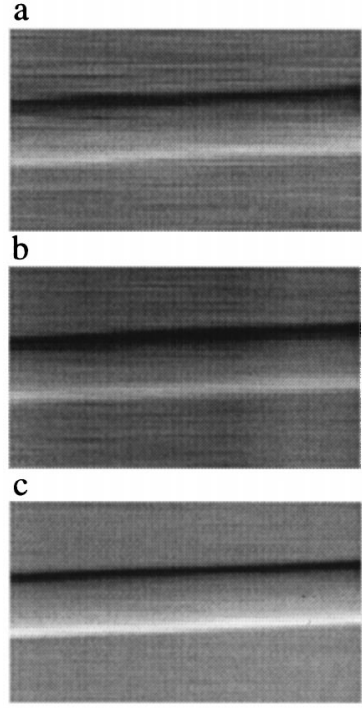

FIG. 7. Images of a $160 \mu \mathrm{m}$ nylon wire obtained at $20 \mathrm{keV}$ with the experimental setup shown in Fig. 6(b). By reducing the beam height from $100 \mu \mathrm{m}$ (a) to 80 and $35 \mu \mathrm{m}$ [(b) and (c), respectively], the contrast increases (see the text).

the copper slit is completely unessential, and could thus be removed provided that the distance between the center of the two beams is increased from 100 to $300 \mu \mathrm{m}$.

The third and last step of the setup development arises from the fact that in most cases, while one of the narrow beams is impinging on a detail, the other one just increases the background, and vice versa. It follows then that even higher signals can be achieved by eliminating one of the two beams, and utilizing a single narrow beam impinging on one of the pixel edges [see Fig. 6(b)]. In this case the symmetry of the signal is clearly broken, but two intense peaks, one positive and one negative, still reveal the presence of a detail within the imaged sample. As it can be seen from the righthand side of Fig. 6(b), in this way a further gain in the signal is achieved, and the fact that the detector pixel dimension has no influence on the acquired signal is even more evident.

Another interesting aspect of this last setup lies in the fact that the narrower the incoming beam, the more intense the acquired signal. In fact, by narrowing the beam, the remaining photons are more easily deviated inside/outside the detector active surface when the sample is scanned through the beam. Thus, their contribution to the signal is much more relevant. Of course the beam intensity is increased accordingly, in order to preserve the number of detected photons per acquisition step avoiding an increase of the quantum noise. In a scanning acquisition modality, this results in a dose increase with respect to absorption imaging proportional to the fraction of the beam impinging outside the detector active surface (this aspect will be discussed in more detail below, when images of the Ackermann phantom will be presented).

Figure 7 shows an experimental demonstration of the signal increase due to beam height reduction. The same object (a $160 \mu \mathrm{m}$ diameter nylon wire) has been imaged at $20 \mathrm{keV}$ with a scanning step of $10 \mu \mathrm{m}$ utilizing a single narrow
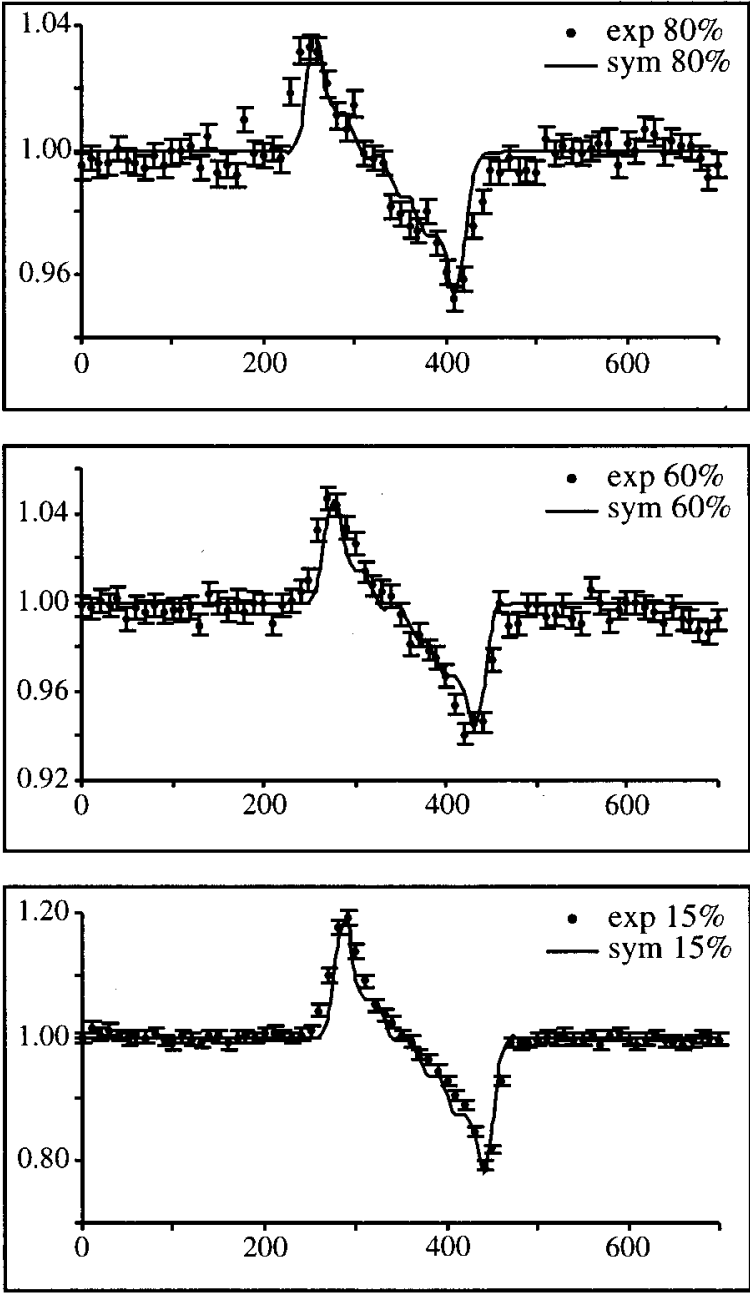

FIG. 8. Experimental data (dots) superimposed on simulated data (solid line) relative to the wires shown in Fig. 7. For all graphs, the relative intensity is given as a function of the displacement in microns. Error bars are equal to the square root of the number of counts (also see Fig. 3).

beam, illuminating 80\% [Fig. 7(a)], 60\% [Fig. 7(b)], and only $15 \%$ [Fig. 7(c)] of the pixel. In all cases, $20 \mu \mathrm{m}$ of the beam thickness fell outside the active surface of the detector (i.e., on the copper mask, since for practical reasons we always left the mask in its position after the careful alignment). The geometrical configuration is thus very similar to the one shown in Fig. 6(b), the only difference being the beam height [which in this case is equal to 100, 80, and $35 \mu \mathrm{m}$ for Figs. $7(\mathrm{a}), 7(\mathrm{~b})$, and $7(\mathrm{c})$, respectively]. From these images the image formation mechanism is immediately apparent: one narrow white line (the positive peak) and a black one (the negative peak) underline the presence of the wire, and the contrast increases as the beam is made narrower.

This is more evident in Fig. 8, where the experimental points (dots with error bars) are superimposed on the simulated signal (solid line). The relative intensity is plotted as a function of the spatial displacement in the scanning direction, and the signal can be directly read out on the vertical axis. From top to bottom, plots correspond to Figs. 7(a), 7(b), and $7(\mathrm{c})$, and the acquired signal is of the order of $9 \%, 11 \%$, 
a

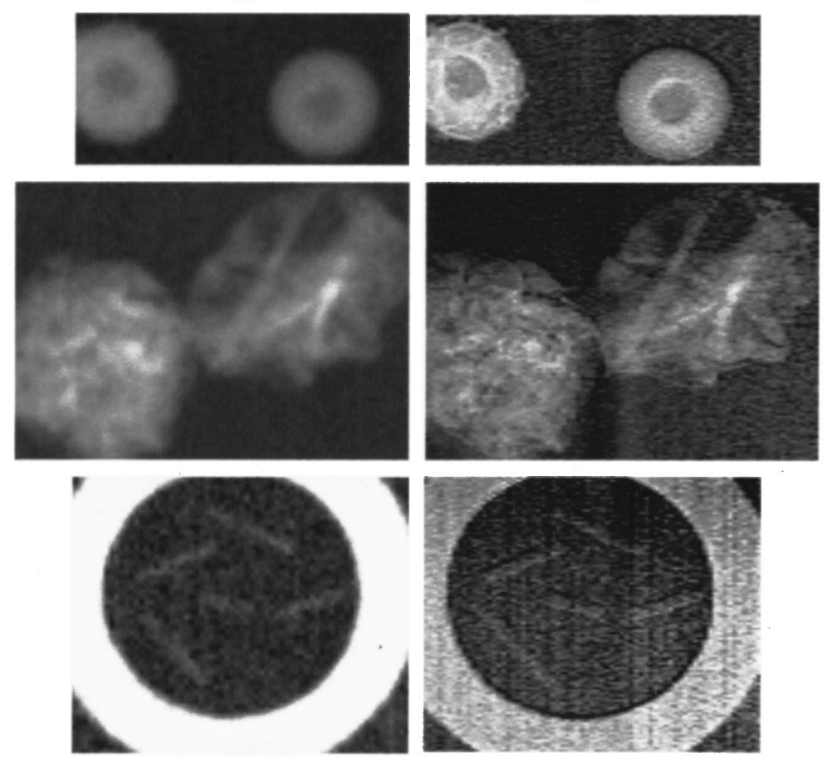

FIG. 9. Images of details from a conventional mammographic test object. (a) is the absorption image and (b) the phase contrast image obtained with the setup shown in Fig. 6(b): the improvement in image quality obtained with this setup is apparent.

and $40 \%$, respectively. It should be noted once again that with pure absorption effects, a $160 \mu \mathrm{m}$ nylon wire at $20 \mathrm{keV}$ would result in a $\sim 0.3 \%$ signal (practically undetectable: for this reason the absorption image is not shown here). This means that with this setup we have obtained a gain in the intensity of the signal (i.e., in the contrast) higher than two orders of magnitude.

It is important to notice that the portion of the beam that impinges within the detector active surface is responsible for all the absorption signal as well as for the negative peaks in the phase contrast signal, while the part of the beam falling outside the detector provides the positive peaks in the phase contrast signal but does not contribute to the absorption signal. Furthermore, as mentioned above, this part of the beam results also in a dose increase. Nevertheless, exploiting also the positive and not only the negative phase contrast peaks practically means doubling the signal in all those cases where absorption is negligible with respect to phase contrast, as clearly visible in Fig. 8.

\section{Application of the technique to a standard mammographic phantom}

The same technique was then applied to a conventional mammographic phantom (Ackermann Phantom, RMI 160, Gammex, Middleton, WI). Figure 9 shows three details extracted from the phantom images: simulated lymphonodes (top), simulated tissue samples (middle), and simulated fibers (bottom, within the ring). A complete description of the phantom can be found in Ref. 32. Figure 9(a) shows the absorption image of the phantom (sample-to-detector distance $\sim 0$ ) and Fig. 9(b) the phase contrast image (sampleto-detector distance $\sim 1.8 \mathrm{~m}$ ) obtained with the setup of Fig. 6(b) (but different beam height). The improvement in image quality obtainable with the proposed setup is apparent. It is important to notice that the "noisy" appearance of Fig. 9(b) is not due to the proposed technique, but to the inner structure of the phantom, which is granular. In other words, the proposed technique depicts correctly the structure in which the details are embedded, and thus this "granularity" should not be interpreted as image noise. This effect was already observed and discussed in Ref. 33.

As already pointed out in a previous work, ${ }^{30}$ the image quality visible in Fig. 9(a) is higher than that one provided by conventional mammographic equipment (images of the same phantom obtained on a conventional equipment can be found in the same reference, as well as in Refs. 10,33). Thus, Fig. 9(b) demonstrates the further improvements in image quality that can be achieved by means of the phase contrast technique and the proposed setup.

Figure 9(a) and Fig. 9(b) were obtained at $22 \mathrm{keV}$ in the same conditions, except for the sample-to-detector distance and beam intensity. In particular, the number of detected photons per detector pixel per acquisition step (i.e., the number of counts per image pixel) was held constant at about 10 000. For Fig. 9(b) (phase contrast), the beam height was equal to $100 \mu \mathrm{m}$, half impinging on the detector active surface and half immediately outside it. For Fig. 9(a) (absorption), the beam height was also equal to $100 \mu \mathrm{m}$, but perfectly matching the detector active surface. For the phase contrast image, only $50 \mu \mathrm{m}$ of the beam were impinging on the detector. Thus, in order to preserve the same overall acquisition time, the beam was made two times more intense (by removing an appropriate absorber) when the phase contrast image was acquired. In this way, by scanning the samples through the beam with exactly the same speed, the same number of photons per detector pixel per unit time is detected. It is thus clear that, when the phase contrast image is acquired, a dose increase of a factor 2 is necessary with respect to the absorption image. In fact, in both cases the sample is scanned through a $100 \mu \mathrm{m}$ high beam with the same speed, but the beam intensity is increased by a factor 2 for the phase contrast image. However, a few things should be noticed at this point.

(1) In Ref. 30, it was demonstrated that absorption imaging with synchrotron radiation and the SYRMEP/ FRONTRAD detector device can be performed while delivering to the sample a Mean Glandular Dose (MGD) of about $0.3 \mathrm{mGy}$, thus reduced by a factor $4-5$ with respect to conventional mammography: this reduction is due to the monochromaticity of the synchrotron beam and to the high efficiency of the detector device. Thus, phase contrast imaging with the proposed setup would be feasible at a dose level reduced by at least a factor of 2 with respect to conventional mammographic equipments.

(2) The dose is increased by a factor of 2 with respect to the absorption image because for practical reasons $50 \mu \mathrm{m}$ of the beam were left outside the detector active surface. One can expect, however, that it would be sufficient to leave a smaller portion of the beam outside the active surface without compromising image quality, thus reducing the delivered 
dose. Investigations are on the way to prove this fact on a wider range of samples.

(3) As indicated by the results shown in Fig. 7 and 8, a further narrowing of the beam would result in higher image contrast. Higher contrast means a higher signal to noise ratio (SNR) at the same dose, or, alternatively, the same SNR at a lower dose. This aspect is also currently under study on a wide range of samples; in this first study the beam was set to $100 \mu \mathrm{m}$ to facilitate the comparison between the two images.

Figure 9 already proves the fact that high gains in image quality can be achieved when typical mammographic objects are imaged, at dose levels lower or comparable to conventional mammographic ones.

\section{E. A comparison with diffraction imaging results}

It should be noted that the results obtained by means of the herein described single narrow beam setup have a strong similitude with another approach to the detection of phase effects, called diffraction (or diffraction enhanced) imaging $^{20,33}$ (compare, for instance, the images of Figs. 7 and 9(b) with diffraction images in Refs. 20 and 33]. In this approach, an analyzer crystal is placed between sample and detector, and the narrow reflectivity curve of the crystal is used to discriminate diffracted from undiffracted photons. In the setup proposed here, by narrowing the beam and shifting it with respect to the center of the pixel, something very similar is realized: one can, in fact, imagine a "box function" that discriminates the photons that are detected from the ones that are not detected (since they do not impinge on the active surface of the detector) according to the diffraction angle they have when they come out of the sample. The relative shift between narrow beam and pixel center determines the position of this "box function" along the angle axis, while the sample-to-detector distance and the pixel height determine its width (the higher the sample-to-detector distance and the smaller the pixel, the narrower the box function). The relevant aspect here lays in the fact that in our case the width of this "box function," to some extent equivalent to the "rocking curve" in diffraction imaging, can be easily tuned by changing the pixel dimensions or, even more easily, the sample-to-detector distance.

\section{F. Scatter imaging}

The final step of the herein described feasibility study consisted in shifting the narrow beam completely out of the detector active surface by very small amounts. In this way, scatter imaging becomes feasible. The setup is very similar to the one shown in Fig. 6(b), with the narrow beam shifted upwards by approximately $15-20 \mu \mathrm{m}$. It is important to notice that, since the sample-to-detector distance is equal to 1.8 $\mathrm{m}$, in this way extremely small scattering angles (a few tenths of microradians) are taken into account. Thus, extremely small angle scatter images are acquired in this way.

Considering the fact that we are using monochromatic radiation, and that only elastic forward scattering is taken into account, the large dimensions of the pixel are an advan-

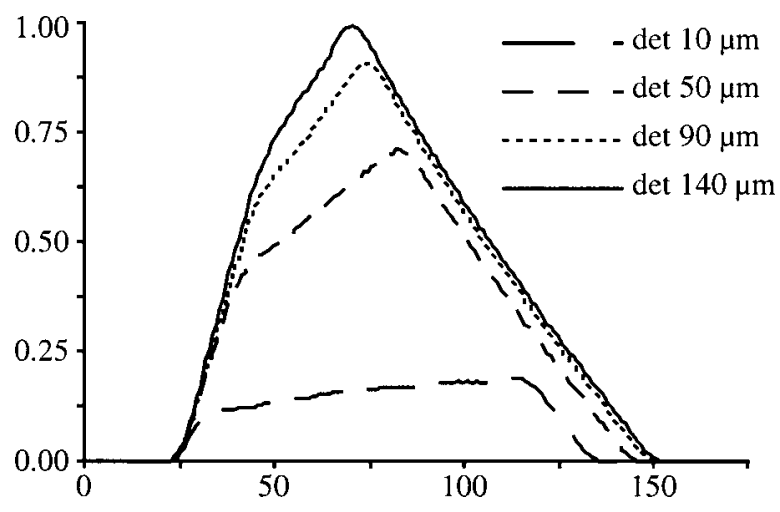

FIG. 10. Influence of the pixel size on extremely small angle scatter imaging. As the pixel size is enlarged, the signal increases until nearly all the scattered photons are collected. The simulated scattered intensity (in arbitrary units) is given as a function of the displacement in microns.

tage rather than a disadvantage in this case: by increasing the pixel vertical dimension, one collects more scattered photons, thus increasing the acquired signal.

In Fig. 10, simulated plots show the behavior of the signal as a function of the pixel size; the simulated sample was a $100 \mu \mathrm{m}$ nylon wire, radiation energy was equal to $20 \mathrm{keV}$. As it can be seen, the scattering peak (that will result in a strong dark halo in the image when the beam impinges on a detail) increases as the pixel size is made larger, until at a certain point nearly all the scattered photons are collected, and the signal is consequently maximized. Since here we are dealing with photons that deviated by a very small amount from their original propagation direction, the shape of the details is somehow preserved in the images, even though only scattered-and not direct-radiation is detected. This fact is demonstrated by Fig. 11, which shows an image of one of the details of the Ackermann phantom (the simulated lymphonodes) obtained only with scattered radiation. As it can be seen, since now scattered photons are added to the background, instead of subtracted as it occurs in conventional absorption imaging, the contrast of the details is reversed with respect to Fig. 9(a).

The last important remark regards the fact that the detection of scattered photons can be achieved at no dose expense by simply adding a further detector layer above (or below, or both above and below) the layer that is acquiring the conventional absorption (or phase contrast, according to the pixel dimensions) image. Investigations are thus now directed toward the optimization of such a multilayer detector

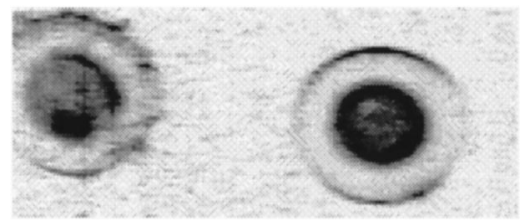

FIG. 11. Image of a detail of the mammographic test object obtained making use only of scattered radiation. The contrast is reversed with respect to absorption imaging [Fig. 9(a)]. 
device: a first three-layer prototype has already been built and successfully tested by our collaboration. ${ }^{13,34}$

\section{CONCLUSIONS}

A research and development study on novel techniques in the field of medical imaging carried out by the SYRMEP/ FRONTRAD collaboration, which involves the use of synchrotron radiation and a novel silicon pixel detector prototype, has been described. The phase contrast imaging technique has been discussed, and its possible implementation in our experimental setup has been described in detail.

In particular, innovative setups based on narrow beams shifted with respect to the center of the detector active surface have been studied, and it has been shown that they allow the possibility of removing the pixel size constraints, thus providing low dose, high quality digital phase contrast images of several samples, also with a relatively large (hundreds of microns) pixel size. Furthermore, the possibility of extremely small angle scatter imaging, and its possible application at no dose expense to diagnostic radiology with synchrotron radiation, has been briefly discussed. Effort is now directed toward the possibility of exploiting all these techniques simultaneously by means of an optimized multilayer detector, thus maximizing the amount of information extracted from the sample in a single acquisition without increasing the delivered dose.

\footnotetext{
a) Corresponding author (olivo@trieste.infn.it)

${ }^{1}$ Mammography - A User's Guide, NCRP Report 85, 1986

${ }^{2}$ H. E. Johns and J. R. Cunningham, The Physics of Radiology, 4th ed. (Thomas, Springfield, IL, 1983).

${ }^{3}$ H. E. Johns and M. J. Yaffe, "X-ray characterization of normal and neoplastic breast tissues," Phys. Med. Biol. 32, 675-695 (1987).

${ }^{4}$ X. Zhou and R. Gordon, "Detection of early breast cancer: an overview and future prospects," Crit. Rev. Biomed. Eng. 17, 203-255 (1989).

${ }^{5}$ L. W. Basset, "Mammographic analysis of calcifications," Radiol. Clin. North Am. 30, 93-105 (1992).

${ }^{6}$ M. Sabel and H. Aichinger, "Recent developments in breast imaging," Phys. Med. Biol. 41, 315-368 (1996).

${ }^{7}$ F. Arfelli et al., "SYRMEP (SYnchrotron Radiation for MEdical Physics). Performance of the digital detector system," Phys. Medica IX, 229233 (1993).

${ }^{8} \mathrm{~F}$. Arfelli et al., "Silicon x-ray detectors for synchrotron radiation digital radiology," Nucl. Instrum. Methods Phys. Res. A 353, 366-370 (1994).

${ }^{9}$ F. Arfelli et al., "An edge-on silicon strip detector for x-ray imaging," IEEE Trans. Nucl. Sci. 44, 874-880 (1997).

${ }^{10} \mathrm{~F}$. Arfelli et al., "A linear array silicon pixel detector: images of a mammographic test object and evaluation of the delivered doses," Phys. Med. Biol. 42, 1565-1573 (1997).

${ }^{11}$ E. Beuville et al., "An application specific integrated circuit and data
}

acquisition system for digital X-ray imaging," Nucl. Instrum. Methods Phys. Res. A 406, 337-342 (1998).

${ }^{12}$ F. Arfelli et al., "Silicon detectors for digital radiography," Nucl. Instrum. Methods Phys. Res. A 367, 48-53 (1995).

${ }^{13}$ F. Arfelli et al., "A multi-layer silicon microstrip detector for single photon counting digital mammography," Informacije MIDEM 29, 26-31 (1999).

${ }^{14}$ F. Arfelli et al., "Improvements in the field of radiological imaging at the SYRMEP beamline," SPIE Vol. 3770, 1999.

${ }^{15}$ A. Snigirev, I. Snigireva, V. Kohn, S. Kuznetsov, and I. Scelokov, "On the possibilities of X-ray phase contrast microimaging by coherent highenergy synchrotron radiation," Rev. Sci. Instrum. 66, 5486-5492 (1995).

${ }^{16}$ T. J. Davis, D. Gao, T. E. Gureyev, A. W. Stevenson, and S. W. Wilkins, "Phase-contrast imaging of weakly absorbing materials using hard X-rays," Nature (London) 373, 595-598 (1995).

${ }^{17}$ P. Cloetens, R. Barrett, J. Baruchel, J. P. Guigay, and M. Schlenker, "Phase objects in synchrotron radiation hard X-ray imaging," J. Phys. D 29, 133-146 (1996).

${ }^{18}$ T. Takeda, A. Momose, Y. Itai, J. Wu, and K. Hirano, "Phase contrast imaging with synchrotron radiation $\mathrm{X}$-rays for detecting cancer lesions," Acad. Radiol. 2, 799-803 (1995).

${ }^{19}$ V. S. Gerasimov et al., "Search for biological objects by refraction radiography using synchrotron radiation of VEPP-3 storage ring," Nucl. Instrum. Methods Phys. Res. A 405, 525-531 (1998).

${ }^{20}$ D. Chapman et al., "Diffraction enhanced X-ray imaging," Phys. Med. Biol. 42, 2015-2025 (1997).

${ }^{21} \mathrm{M}$. DiMichiel et al., "Phase contrast imaging in the field of mammography," in Medical Applications of Synchrotron Radiation, edited by M. Ando and C. Uyama (Springer-Verlag Tokyo, 1998), pp. 78-82.

${ }^{22} \mathrm{~F}$. Arfelli et al., "Low-dose phase contrast X-ray medical imaging," Phys. Med. Biol. 43, 2845-2852 (1998).

${ }^{23} \mathrm{E}$. A. Beliaevskaya et al., "X-ray imaging of a synthetic mammography structure," Phys. Medica XIV, 19-23 (1988).

${ }^{24}$ V. N. Ingal, E. A. Beliaevskaya, A. P. Brianskaya, and R. D. Merkurieva, "Phase mammography—a new technique for breast investigation," Phys. Med. Biol. 43, 2555-2567 (1988).

${ }^{25}$ M. Born and E. Wolf, Principles of Optics (Pergamon, Oxford, 1975).

${ }^{26}$ J. Cowley, Diffraction Physics (North Holland, Amsterdam, 1975).

${ }^{27}$ S. W. Wilkins, T. E. Gurejev, D. Gao, A. Pogany, and A. W. Stevenson, "Phase contrast imaging using polychromatic hard X-rays," Nature (London) 384, 335-338 (1996).

${ }^{28} \mathrm{~F}$. Arfelli et al., "Digital mammography with synchrotron radiation," Rev. Sci. Instrum. 66, 1325-1328 (1995).

${ }^{29}$ F. Arfelli et al., "The mammography project at Elettra," Phys. Medica XIII, 7-12 (1997).

${ }^{30} \mathrm{~F}$. Arfelli et al., "Mammography of a phantom and breast tissue with synchrotron radiation and a linear-array silicon detector," Radiology 208, 709-715 (1998).

${ }^{31}$ A. Olivo, "Un metodo innovativo per produrre radiografie digitali in contrasto di fase con luce di sincrotrone," Ph.D. dissertation, University of Trieste, 1999, unpublished.

${ }^{32}$ J. Law, "A new phantom for mammography," Br. J. Radiol. 64, 116-120 (1991).

${ }^{33} \mathrm{~F}$. Arfelli et al., "Mammography with synchrotron radiation: phasedetection techniques," Radiology 215, 286-293 (2000).

${ }^{34} \mathrm{~F}$. Arfelli et al., "A multilayer edge-on silicon microstrip single photon counting detector for digital mammography," Nucl. Phys. B 78, 592-597 (1999). 